\title{
Beam gap transient analysis and mitigations in high-current storage rings for an electron-ion collider
}

\author{
T. Mastoridis (-)" \\ California Polytechnic State University, 1 Grand Ave., San Luis Obispo, CA 93407, USA \\ J. D. Fox (우 \\ Stanford University, 450 Serra Mall, Stanford, CA 94305, USA \\ J. Guo, R. A. Rimmer, and H. Wang \\ Jefferson Lab, 12000 Jefferson Avenue, Newport News, VA 23606, USA
}

(Received 5 February 2020; accepted 25 September 2020; published 12 October 2020)

\begin{abstract}
The U.S. electron ion collider will utilize high current electron and ion storage rings with many bunches and large rf systems. Because of the dissimilarity of the two rings, the rf transients created by gaps or variations in the current distributions will be very different in the two rings. These transients cause a shift in the synchronous phase of the beams as a function of rf bucket position, can impact the luminosity through shifts in longitudinal position of the IP, will affect the performance of the rf and LLRF control loops, and may require significant rf power overhead to control. A machine design that uses superconducting crab cavities will also have sensitivity to gap transients and synchronous phase variations along the bunch train with variations in crab cavity voltage seen by each bunch, since the high $Q$ of the crab cavities precludes modulating them to compensate for the time of arrival shifts caused by the gap transients in the main rf systems. All these effects make the problem of managing gap transients crucial to the operation of the EIC. This work presents methods to study the dynamics of the rf and LLRF systems for these heavily beam loaded facilities. An illustrative machine design example is presented and used to investigate the expected magnitudes of the rf gap transients, and exploration of various possible remedies to match the gap transients in the two dissimilar EIC rings. In addition to the study of the power required and gap transients, this work also estimates longitudinal coupled-bunch instabilities due to the baseline cavity fundamental impedance. The work is motivated to emphasize the importance of tools and methods to estimate these effects as part of the early design phase of the Electron-Ion Collider or any high current storage ring design.
\end{abstract}

DOI: 10.1103/PhysRevAccelBeams.23.101601

\section{METHODOLOGY}

The design and operation of heavily beam loaded circular accelerators requires care in the design and set up of the rf accelerating systems, and often requires complex LLRF systems to manage the stability of the beam as well of the rf system itself [1-3]. These methods usually use feedback techniques to regulate the cavity fields and to control the interaction of the machine impedances with the circulating beam [4-6]. Typical fill patterns require gaps in the populated bunches for ion clearing or mitigation of electron-cloud effects or for practical injection/extraction kicker systems. These beam gaps give rise to periodic

\footnotetext{
*tmastori@calpoly.edu
}

Published by the American Physical Society under the terms of the Creative Commons Attribution 4.0 International license. Further distribution of this work must maintain attribution to the author(s) and the published article's title, journal citation, and DOI. transients in the rf system. For collider facilities, the management of these gaps and gap transients has great impact on the matching of the synchronous phases of the intersecting bunches, which impacts the $z$ location of the luminous region. This in turn can cause luminosity loss depending on the IP lattice details. Several of the new facility designs incorporate crab cavities as a method to level or increase luminosity, and the gap transients, with associated shifts in synchronous phase, can also have very significant impacts on the effectiveness of the crab cavity systems.

In this work we study a strawman EIC machine design using time domain and frequency domain simulation methods. These tools have been developed to be easily adaptable to various EIC machine designs, and our motivation is to highlight important design choices and challenges in the rf and LLRF systems. This example design study is based on a parameter set from the highest energy Jefferson Lab EIC design proposal: a $10 \mathrm{GeV}$ electron ring 
and a $200 \mathrm{GeV}$ hadron ring [7-9]. This illustrative case shows the techniques and methods that are applicable to the general class of high beam current colliders using both superconducting and normal rf technology. The resulting models have been used to explore and evaluate a range of possible mitigation techniques for the gap transient asymmetry and develop tools to estimate coupled-bunch instabilities driven by the cavity fundamental impedance. Because the EIC must operate over a wide range of center of mass energies there will be many other cases being proposed, and they too will need to be estimated and evaluated as part of the EIC machine design studies.

The coupling of the beams and the rf systems is nonlinear, and the technical implementations of the LLRF regulating loops often have nonlinear behavior, such as klystron saturation, etc. To study, model and optimize these LLRF, rf and beam systems several simulation methods have been developed. In this work we use a time-domain simulation method that was originally developed for PEP-II [10-12] and subsequently expanded for high-current operation in PEP-II $[13,14]$, and study of the LHC and HL-LHC machines [15].

To evaluate possible gap transient effects, and the impact of LLRF and rf system design choices and technical characteristics, we use a time-domain simulation that has block models representing the rf cavities, rf power stages, the essential LLRF system loops, and a dynamic description of the bunches (determined by the ring lattice momentum compaction, the effective rf cavity voltage as sampled by the bunch and the equations of synchrotron motion). As this is a time domain simulation, it is possible to include nonlinear behavior of a power stage, to include harmonic cavities, to implement limiters or saturating elements in the LLRF systems, or to include other system elements that might be in a practical system. For this work the simulation sub-blocks and topology models have been adjusted to model the EIC rings and possible rf and LLRF technology. The rf systems are represented by macrocavities, so that $\mathrm{N}$ identical rf stations can be represented by 1 macrocavity with $\mathrm{N}$ times the rf voltage. If there are stations with different configurations (e.g., 2 cavities per klystron and also 4 cavities per klystron) each type of $\mathrm{rf}$ station configuration requires a separate macrocavity system. Similarly, if there are harmonic cavities, they must be included to represent the actual total voltage seen by the beam. In the simulation, all of the rf elements are linked through a common beam current represented by macrobunches. The number of macrobunches is a simulation parameter selected to have adequate frequency domain representation of the effects possible in the system bandwidth, e.g., for studies of low frequency beam modes the beam can be modeled by a reduced number of macrobunches. For this EIC study of gap transient effects the ring beam pattern is replicated exactly (i.e., the number of macrobunches is equal to the number of planned bunches in the EIC), to achieve an accurate representation of the gap transient system behavior.

Three different strategies were investigated to mitigate gap transients in this EIC scenario: a "traditional" LLRF feedback system (optionally including a one-turn feedback and/or a feedforward); local bunch intensity modulation by the introduction of double intensity bunches at the beginning or end of the bunch train; and a modulation of the cavity voltage reference. They are respectively described in Secs. II-IV. The resulting rf power requirements and interaction point (IP) time-shift are used as performance metrics to compare these schemes. Section V presents estimates of the longitudinal coupled-bunch instabilities. Section VI explores the impact of physical facilities with large separations between rf power stages and the beam line cavities, which results in longer round-trip signal delays in feedback signal paths. Finally, Section VII presents potential complications due to non-ideal behavior of components and suggests necessary future work as part of further development of the EIC rf systems. A verification of the models and simulations is included in Appendix.

Table I shows the ring and rf system parameters used in this study, including the particle energy $E_{o}$, the total gap voltage $V_{\text {tot }}$, the cavity loaded quality factor $Q_{L}$, the bunch length $\sigma_{z}$, and the average beam current $I_{D C}$.

\section{LLRF FEEDBACK}

\section{A. Direct feedback loop}

Figure 1 presents a block diagram of a possible LLRF and rf system topology that implements four possible feedback paths between the cavity system, the beam, and the LLRF processing through the high power klystron. The path labeled "direct feedback" represents a low bandwidth integrator which sets overall DC operating points, as well as a wide band loop which counteracts the voltages generated by the beam current in the cavity impedance, effectively reducing the impedance seen by the beam. The "one-turn feedback" is a periodic comb filter with high gain at the revolution harmonics, which acts to reduce the impedance at the synchrotron sidebands around the revolution harmonics within the direct loop bandwidth.

TABLE I. Example ring and rf system parameters used in this study.

\begin{tabular}{lccccccccccc}
\hline \hline Ring & $E_{o}(\mathrm{GeV})$ & $V_{\text {tot }}(\mathrm{MV})$ & $N_{\text {cavities }}$ & $R / Q$ & $Q_{o}$ & $Q_{L}$ & $f_{\text {rf }}(\mathrm{MHz})$ & $f_{\text {rev }}(\mathrm{kHz})$ & $\sigma_{z}(\mathrm{~mm})$ & $I_{D C}(\mathrm{~A})$ & $N_{\text {bunches }}$ \\
\hline $\mathrm{e}-$ & 10 & 19 & 24 & 217 & $31 \times 10^{3}$ & 5170 & 476.4 & 128.3 & 13.7 & 0.26 & 864 \\
Ion & 200 & 57.6 & 24 & 208 & $8 \times 10^{9}$ & 28600 & 952.8 & 128.3 & 32 & 0.75 & 864 \\
\hline \hline
\end{tabular}




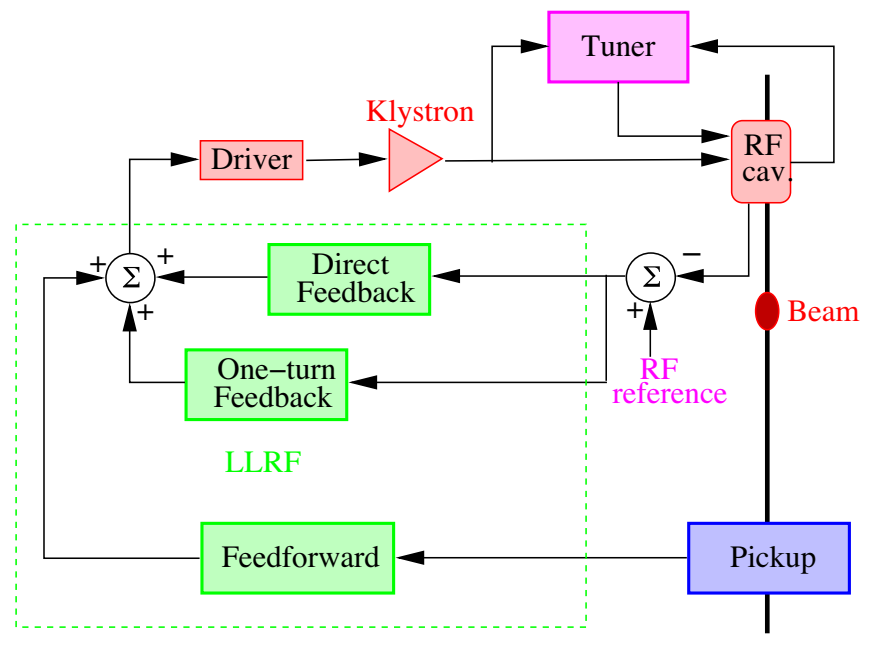

FIG. 1. Block diagram of the topology of the rf and LLRF system studied.

Finally, a "feedforward" path is shown, which can use a beam current signal derived from a pickup to generate a correction signal within the loop. Choosing which of these techniques to implement, and what effectiveness, or drawbacks each brings, is the task of the LLRF and $\mathrm{rf}$ system designers.

To highlight these options, we first implement a simple LLRF system model with a loop delay of 320 ns. This delay estimate is consistent with a facility where the rf power systems are located close to the beam line components. A direct feedback loop is modeled (Fig.e 1), this is a broadband feedback path. The direct loop gain and phase are set to achieve a $10 \mathrm{~dB}$ gain margin and at least $45^{\circ}$ phase margin. The direct loop acts to lower the cavity impedance seen by the beam, much in the same way that feedback around an amplifier reduces the output impedance. In this first study a linear and unsaturated klystron is simulated.

The gain of the loop is limited by the total loop delaybandwidth product $[6,16]$ and the necessary gain and phase margins for stability of the direct loop. The loop bandwidth is $\approx 500 \mathrm{kHz}$. These simulations use $320 \mathrm{~ns}$ total loop delay, which reflects achievable delays if the klystron power stages and cavity systems are located with approximately 24 meters (one-way) of waveguide/cable separation, a klystron group delay of $100 \mathrm{~ns}$ and a signal processing group delay of $20 \mathrm{~ns}$. Waveguide and cable delays can be significant if the rf power stages are located remotely from the rf cavities on the beam line. The PEP-II implementation had 460 ns loop delay, while the LHC implementations achieved $650 \mathrm{~ns}$ loop delay. It will be an important aspect of the EIC rf system design to understand the tradeoffs in locations of klystron power stages, support equipment, and the cavity systems in the machine tunnel against the performance of the LLRF impedance control. These effects are explored in Sec. VI.

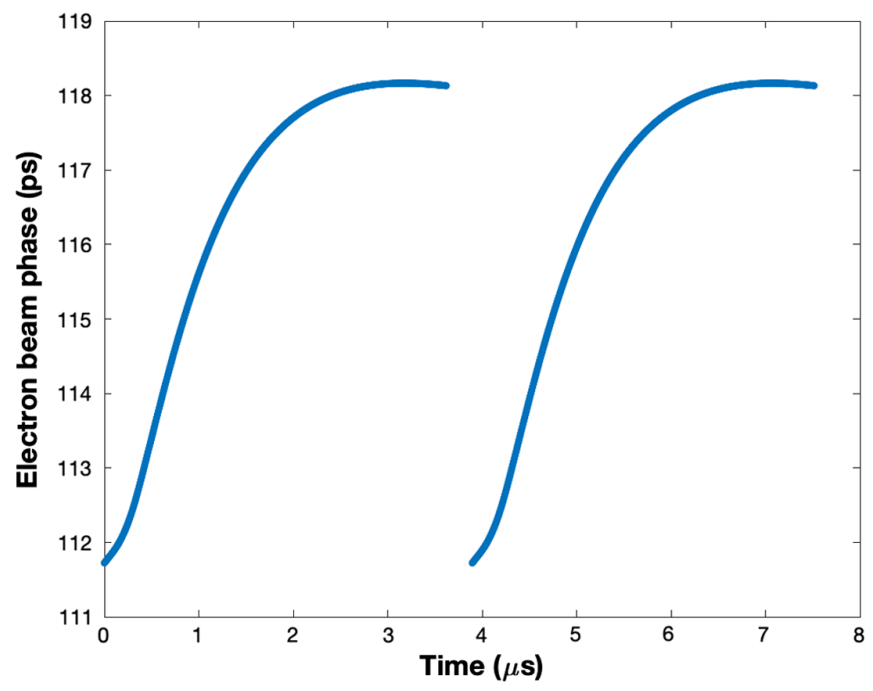

FIG. 2. Electron beam synchronous phase transient.

Figures 2 and 3 show the simulation results of the beam synchronous phase transient in the electron ring and ion ring for the baseline direct loop case. The beam pattern consists of two bunch trains of 432 bunches each, with two gaps (269 ns long each). Every fourth bucket is filled in the electron ring and every eight in the ion ring (bunch spacing of $8.4 \mathrm{~ns}$ ). In these examples the rf cavity detuning has been selected to minimize the average (over a turn) rf reflected power.

The time offset at the IP is defined as the difference in arrival times of the colliding bunches, which equals the difference between the transients in the two rings. The time offset can be converted to a z-vertex offset by multiplying by $c / 2$. The IP time offset is seen in Fig. 4, and shows a peak-to-peak value of $2.4 \mathrm{ps}$ and a standard deviation of $0.82 \mathrm{ps}$ ( 1 degree at $476 \mathrm{MHz}$ is about $6 \mathrm{ps}$ ). Note that there is a nonzero mean value. Since the LLRF cannot perfectly

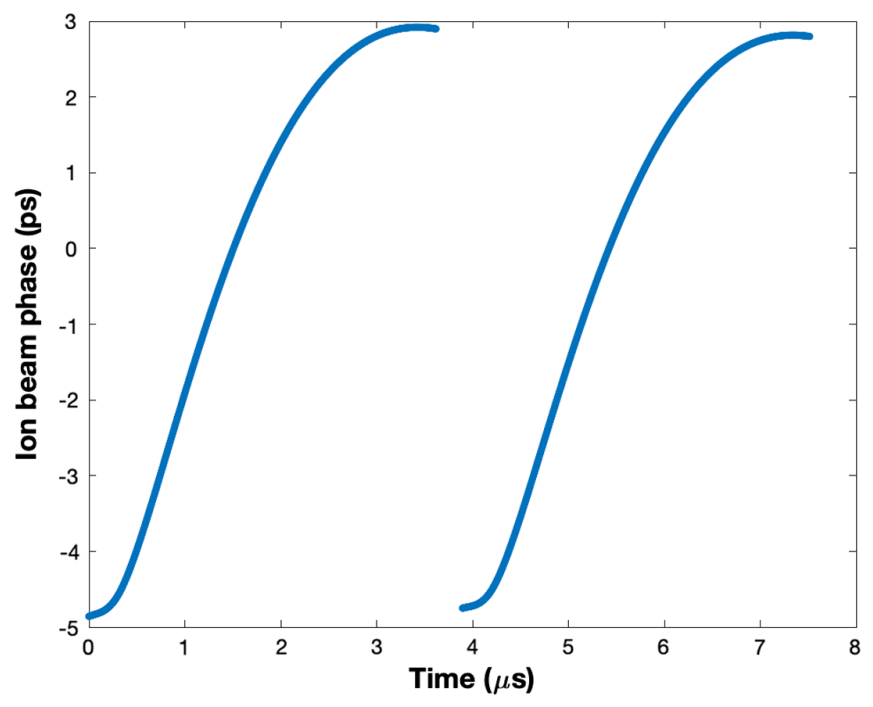

FIG. 3. Ion beam synchronous phase transient. 


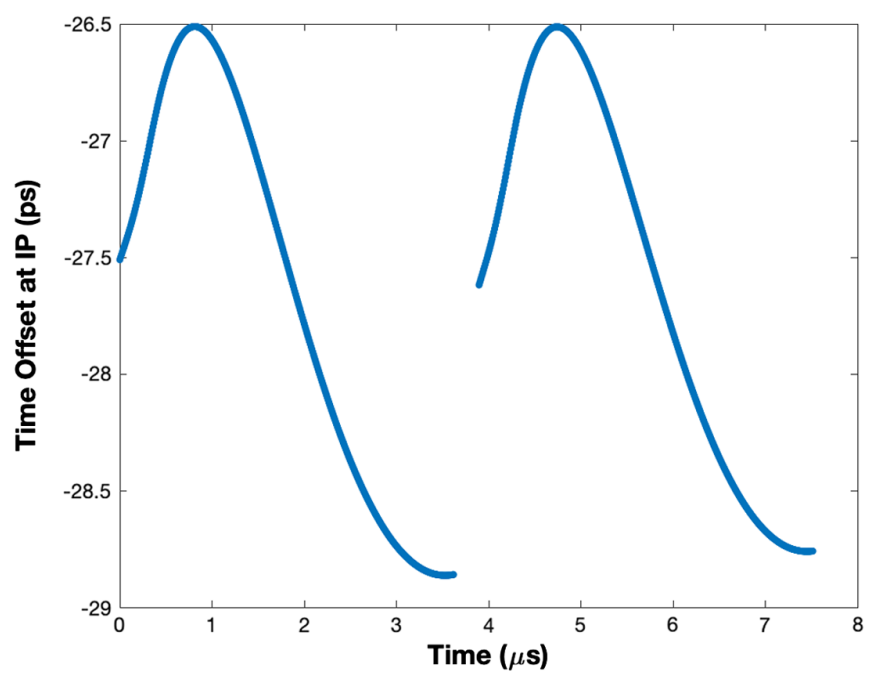

FIG. 4. IP time offset with direct loop feedback.

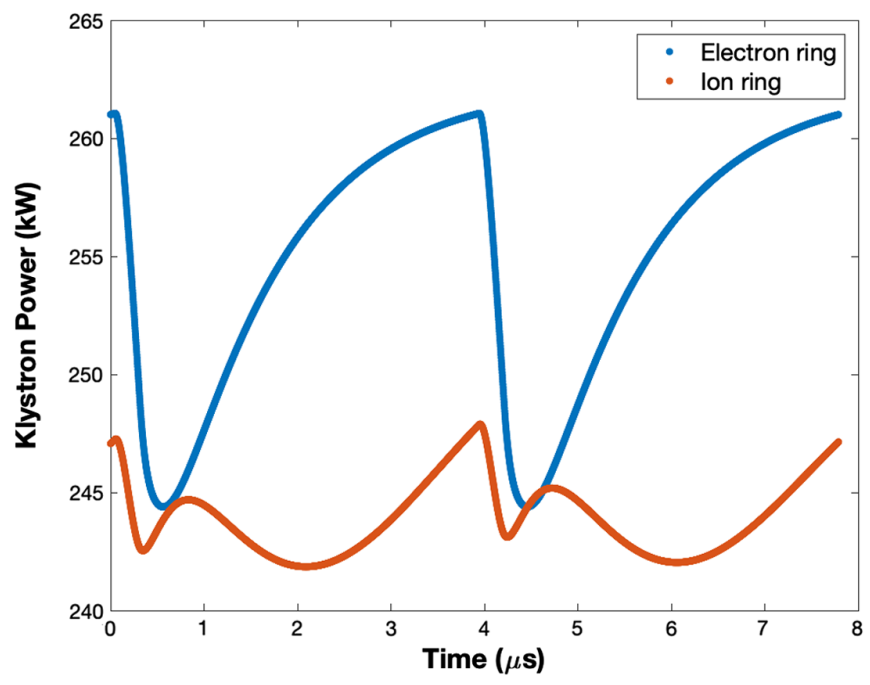

FIG. 5. Klystron power with direct loop feedback.

compensate the beam loading, the mean beam phase slightly differs from the stable phase for each ring. The required klystron power for this case is shown in Fig. 5.

\section{B. One-turn feedback loop}

This base case can be improved through the use of another LLRF feedback loop. As seen in Fig. 1, a one-turn feedback (OTFB, also called a comb loop) system is added to improve performance. This loop is a periodic structure that implements gain at multiples of the revolution frequency, acting to reduce the impedance seen by the beam near the revolution frequency sidebands around the rf fundamental. The OTFB has a gain of 10 at the revolution sidebands and a two-sided bandwidth of $1.3 \mathrm{kHz}$. Figures 6 and 7 present the electron and ion ring gap transients for these cases, The difference between the ring synchronous phases is shown in Fig. 8. The IP shift peak-to-peak time

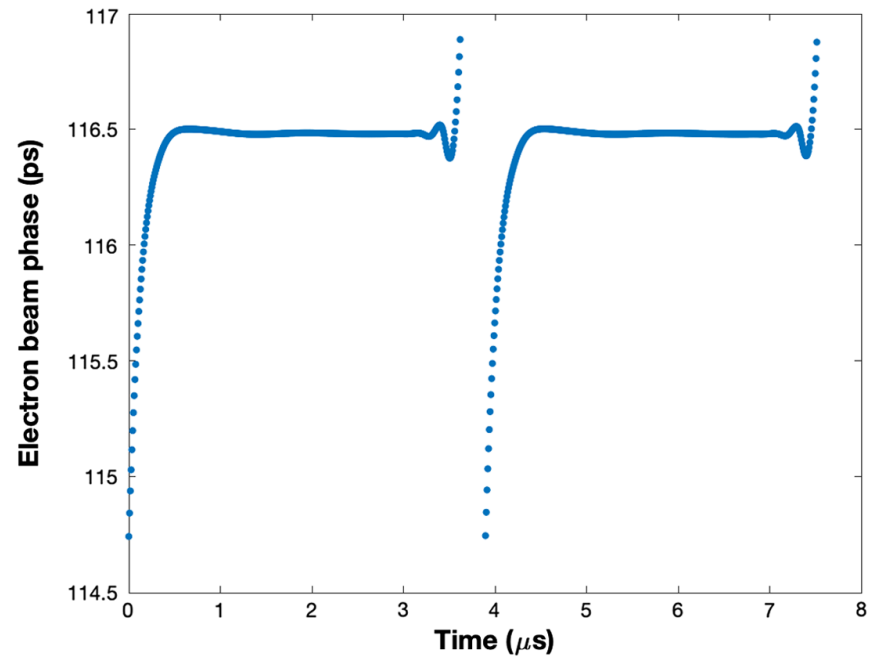

FIG. 6. Electron beam synchronous phase transient.

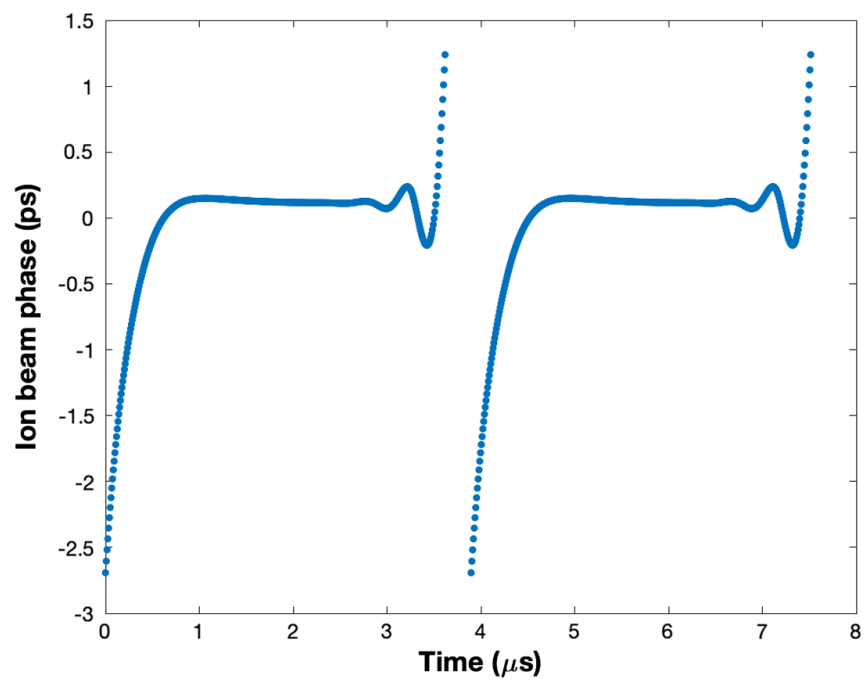

FIG. 7. Ion beam synchronous phase transient.

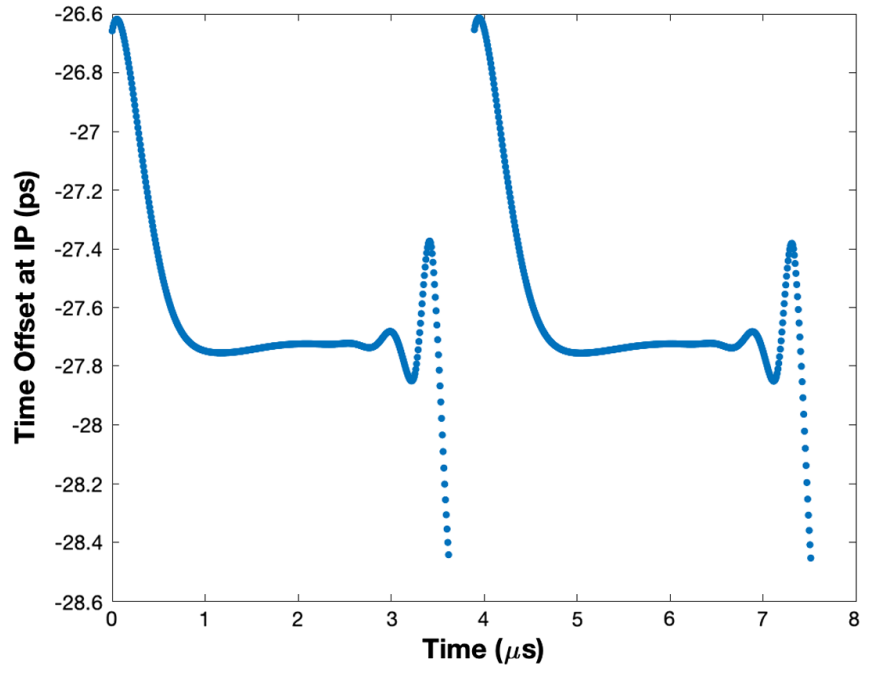

FIG. 8. IP time offset with one-turn feedback. 


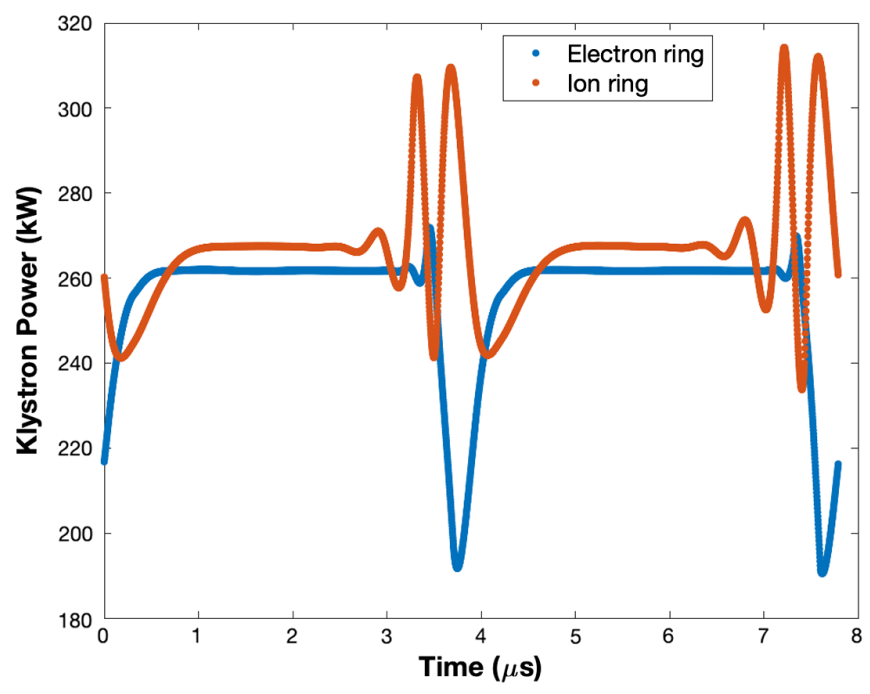

FIG. 9. Klystron power with one-turn feedback.

offset has been reduced to $1.8 \mathrm{ps}$ and the standard deviation to $0.31 \mathrm{ps}$. The required rf powers are shown in Fig. 9. As expected, there is a tradeoff between beam transient reduction and transient klystron power.

These results are for a LLRF and rf system with $320 \mathrm{~ns}$ group delay and the LLRF loop phase and gain optimized for transient response and stability. The klystron model in these cases is ideal, with infinite bandwidth. A realistic klystron bandwidth of $4 \mathrm{MHz}$ was also evaluated in the simulation to evaluate any negative effects. Introducing the klystron bandwidth does not change the performance of the direct and one-turn feedback systems. This is expected, since the beam transient is already filtered by the closed loop cavity response, which has a much smaller bandwidth $(\approx 500 \mathrm{kHz})$ than the klystron.

\section{Feedforward}

Similar to the rf feedback, this technique injects a correction (error) signal into the LLRF summing node before the klystron input $[6,17]$. This feedfoward signal is derived from a separate measurement of the beam current rather than the cavity voltage. It can thus measure the beam current and compensate the voltage it generates in the cavity directly through the klystron path. The feedforward signal is derived from filtered measurements of the beam current through a suitable longitudinal pickup, sampled, and converted to a modulated signal at the rf frequency. This signal is then delayed 1 turn with phase equalization. The effect is to drive a transient through the rf power stage that cancels the beam current component generated in the cavity. The direct and OTFB loops still function for signals within the closedloop bandwidth, providing impedance control at the synchrotron sidebands around the revolution frequencies.

An ideal feedforward system would perfectly cancel the beam transient, behavior that is confirmed in the simulations. However a realistic feedforward system will

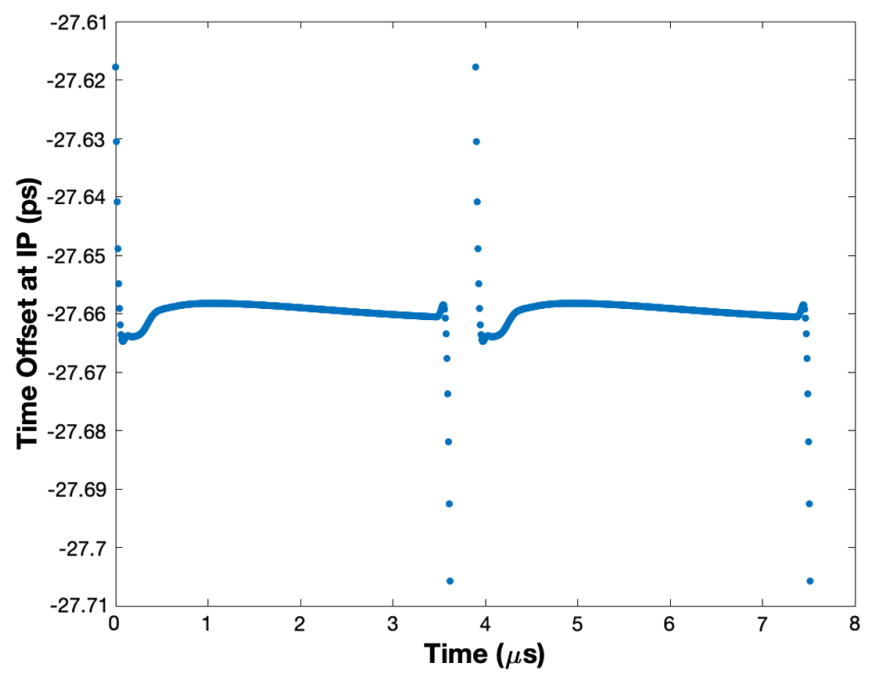

FIG. 10. IP time offset with feedforward.

be limited by the klystron bandwidth and the beam pickup signal to noise ratio. Initial simulations for the EIC example show that even with a 5\% uncertainty error on the demodulated in-phase and quadrature components of the beam pickup signal, the resulting transient has a peakto-peak time offset of about $0.42 \mathrm{ps}$ and a standard deviation of about $0.10 \mathrm{ps}$.

Applying the $4 \mathrm{MHz}$ klystron bandwidth slightly reduces the performance and introduces very small transients in the klystron power (Figs. 10 and 11). The peak-to-peak time offset is now 0.09 ps (dominated by the initial and final transients) and the standard deviation is less than $0.01 \mathrm{ps}$.

\section{Summary of direct, one-turn delay, and feedforward implementations}

Table II summarizes the results for the various LLRF architectures. The simple direct feedback loop achieves acceptable performance. The rms offset at the IP is

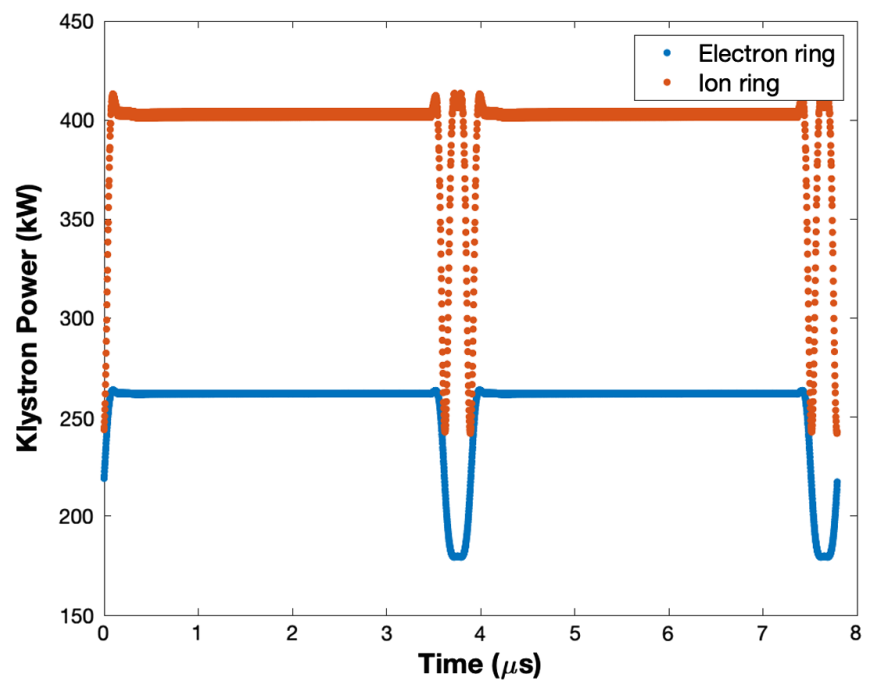

FIG. 11. Klystron power with feedforward. 
TABLE II. IP offset and klystron power for various LLRF configurations. Direct loop is on for all four cases. FF is off for the OTFB case. OTFB is off for the FF cases.

\begin{tabular}{lcccc}
\hline \hline & $\begin{array}{c}\text { IP offset } \\
\text { pk-pk } \\
(\mathrm{ps})\end{array}$ & $\begin{array}{c}\text { IP offset } \\
\sigma(\mathrm{ps})\end{array}$ & $\begin{array}{c}\text { Peak power } \\
\text { electrons } \\
(\mathrm{kW})\end{array}$ & $\begin{array}{c}\text { Peak power } \\
\text { ions (kW) }\end{array}$ \\
\hline $\begin{array}{l}\text { Realistic } \\
\quad \text { Delay }\end{array}$ & 2.4 & 0.82 & 261 & 248 \\
$\begin{array}{l}\text { OTFB } \\
\begin{array}{l}\text { FF with } \\
\text { pickup noise }\end{array}\end{array}$ & 1.8 & 0.31 & 272 & 314 \\
$\begin{array}{l}\text { FF with 4 MHz } \\
\text { klystron BW }\end{array}$ & 0.09 & 0.01 & 264 & $>500$ \\
\hline \hline
\end{tabular}

improved by more than a factor of two with the addition of the OTFB. Using a feedforward system instead of the OTFB leads to even higher performance.

The cost is of course peak klystron power, since sharper transients are required. In the electron ring case, the power does not change much, since the cavity loaded quality factor $Q_{L}$ is low and about half of the power goes to the beam $(131 \mathrm{~kW})$. In the ion case, there is much higher sensitivity to sharp transients and the peak power increases significantly.

For the final EIC design, it will be necessary to establish peak-to-peak and rms IP time offset specifications for any proposed interaction region design. It will then be possible to assess the effectiveness of the various approaches.

\section{BUNCH INTENSITY MODULATION, E.G., DOUBLE INTENSITY BUNCHES}

Another method that has been explored is to adjust the stored charge adjacent to the gaps to try to minimize the gap transient, or to give it a particular shape. A "fill pattern modulation" scheme was suggested for the ALS by John Byrd et al. in 2002 [18]. Double intensity bunches can be injected before or after the gap, so that the average current within the cavity bandwidth is the same throughout the ring. D. Teytelman evaluated this approach and measured the phase transients in the ALS and in BEPC-II with such a modulated fill pattern $[19,20]$.

The suggested beam pattern for the EIC ion ring is shown in Fig. 12. The first 31 bunches on each train have double the intensity.

As expected, there is significant improvement with this scheme. For the scenario with the direct loop on, but without OTFB or feedforward, the rms beam phase is reduced from 2.4 to $0.31 \mathrm{ps}$. The peak-to-peak variation is reduced from 7.6 to $1.1 \mathrm{ps,}$, as shown in Fig. 13. The phase of the double intensity bunches is not shown, since these bunches do not collide and thus do not contribute to luminosity. The initial steep transient corresponds to the double intensity bunches. The performance is almost identical if the double intensity bunches are placed half at the beginning and half at the end of the bunch train.

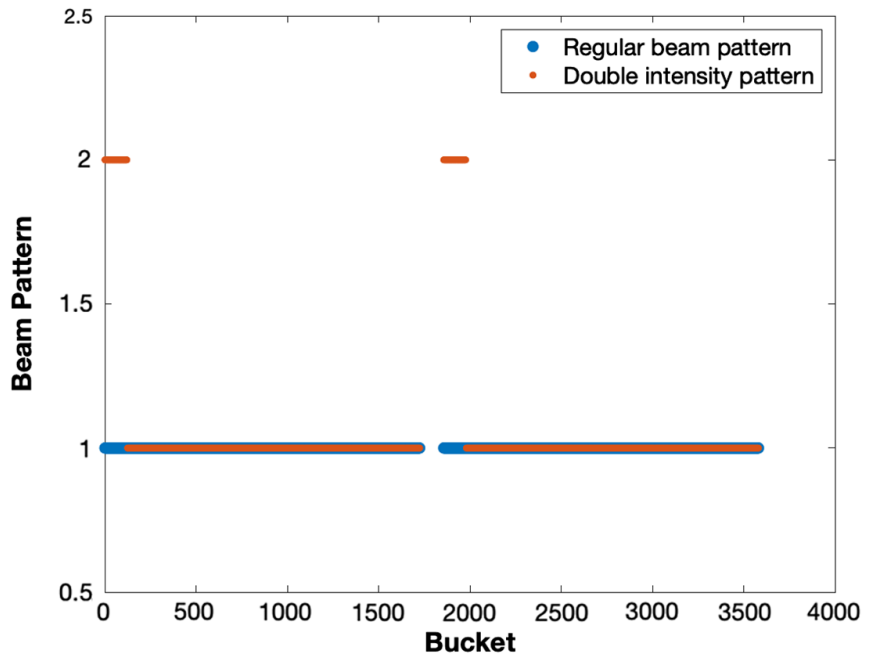

FIG. 12. Nominal and double intensity beam patterns (ion ring).

On the other hand, this scheme cannot work with a OTFB or feedforward system, since the peak power required during the double intensity transient would be enormous and in a practical implementation would result in saturation of the power stages. This is not necessarily a problem if the gap transient reduction through a direct loop is sufficient, so that these additional OTFB or feedforward systems would not be necessary (or helpful).

However the lifetime of these higher intensity bunches, and maintaining the desired current profile, may be challenging from an operational standpoint even with top-up injection. The practical application of this scheme might be limited by beam lifetime from Touschek scattering and other loss mechanisms in the higher intensity bunches. This situation would require top-off to maintain

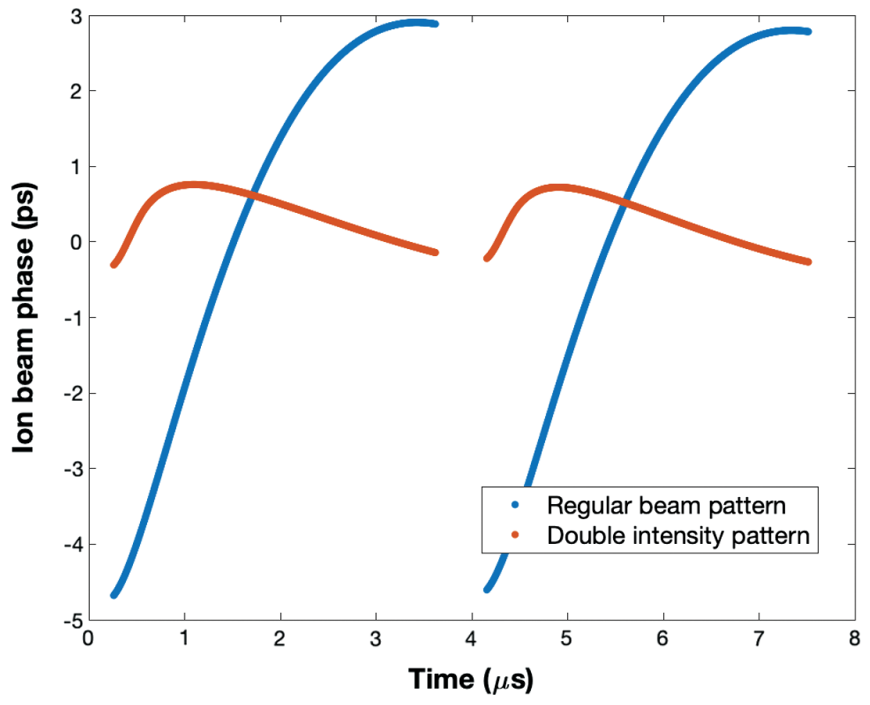

FIG. 13. Beam phase in the presence of double intensity bunches (ion ring). 
fill uniformity for the electron ring, this might not be possible in the ion ring. The double intensity bunches will also have different betatron tunes since they might not interact with the other beam at the collision point (no beambeam effects), or they might be in collision with double intensity bunches in the other ring. It may be very challenging to find operational parameters that will give stable tunes for both the normal and double intensity bunches, especially for the highest current configurations.

\section{A. Masking the error signal}

This scheme can be further improved by masking the cavity error signal (the input to the cavity reference summing node) corresponding to any empty and/or double intensity buckets. This approach, which is a variant of the gap-feedforward method employed in PEP-II [21], keeps the large error signals generated during the gap intervals from saturating the rf power stages. In the PEP-II implementation, the rf reference signals in the gap were modulated via a transient cancellation reference. This periodic transient was computed via a slowly adapting low-pass filtered version of the klystron drive (rejecting the large periodic static error but still providing closed loop AC gain). This approach does not fight the gap transient, it keeps the LLRF and rf systems from saturating trying to correct the gap transient.

For this EIC study, we explore another idea. In this "mask the transient" approach, during the gap interval the feedback error signal is clamped or masked, and the rf drive signal to the klystron comes from the low bandwidth integrator of the LLRF system. Outside of the gap interval, the feedback error signal is applied to the summing node, this error signal path then provides the necessary loop gain for the regulation. In the time domain, this masking is a rectangular multiplication by 1 during the filled portion of the turn, multiplication by zero in the short gap. This is a multiplication by a rect function in the time domain, so in the frequency domain the beam-cavity derived signals are convolved with a sinc weighting function. As the filled bunches are most of the turn, the sinc is narrow in frequency, so it acts to slightly widen the impulselike signals at the revolution harmonic frequencies. In terms on impact on the feedback, there is no rolloff of gain across the operating band.

The figure explores the use of these gap error masking for a case of the ion ring with 31 double intensity bunches in the beginning of each bunch train, with the nominal direct loop gain applied outside of the gap interval. As seen in the figure, the performance is much improved with respect to maximum klystron power and variation of synchronous phase. The beam phase is now essentially constant (less than 0.01 degrees rms). Figure 14 shows the resulting cavity phase. The klystron power is now also effectively constant at $245 \mathrm{~kW}$, as seen in Fig. 15.

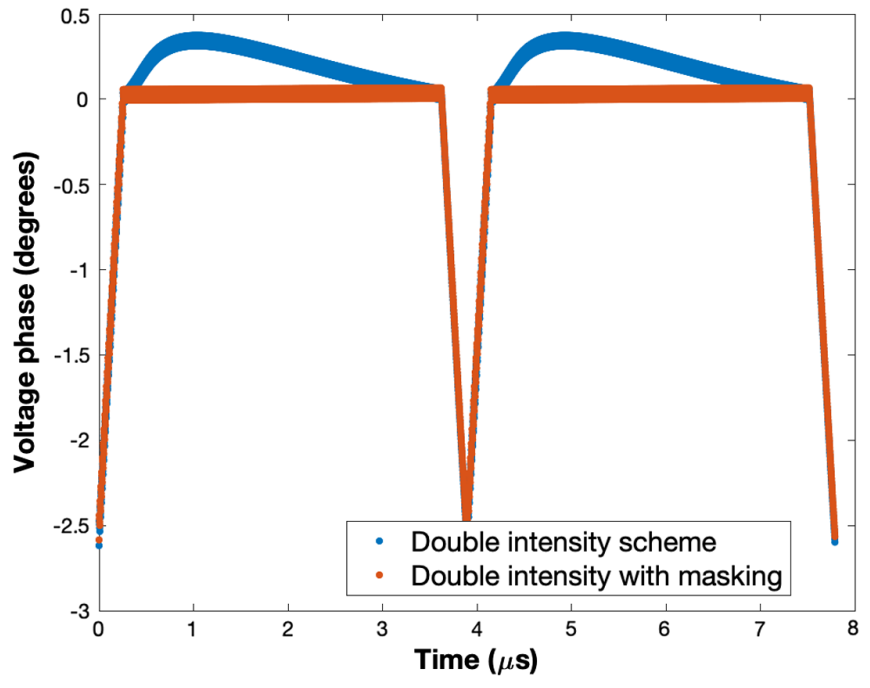

FIG. 14. Cavity phase voltage with and without error signal masking.

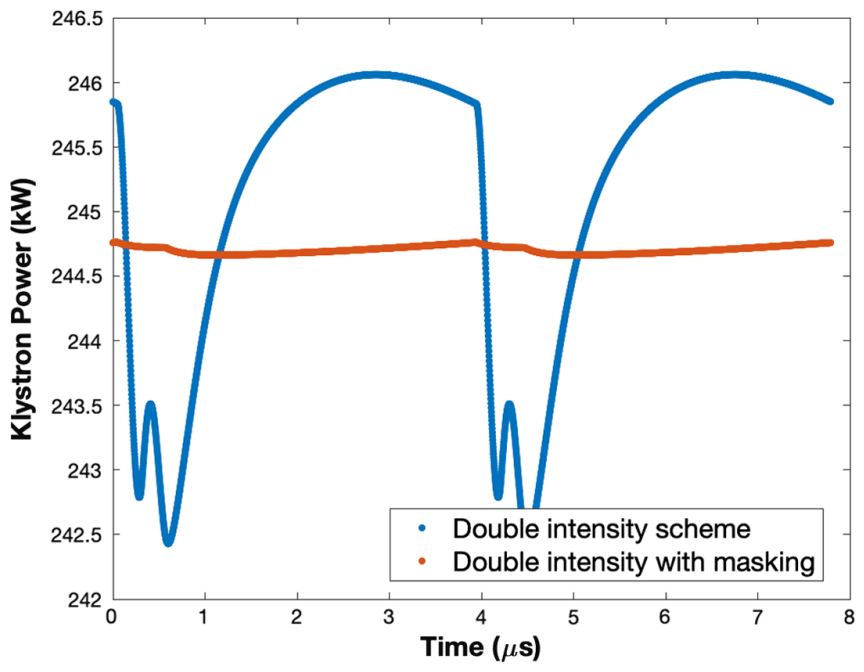

FIG. 15. Klystron output power with and without error signal masking.

\section{CAVITY REFERENCE PHASE MODULATION}

Another approach to minimize the differential IP shift from variations in synchronous phase due to gap transient effects is to modulate a cavity phase reference signal to drive the gap transient shape to better match the transient in the other ring. In [22], it was shown that the cavity reference voltage $V_{\text {ref }}$ could be modulated to minimize klystron power (the cavity voltage $V_{\text {cav }}$ and klystron current $I_{g}$ have the same phase). A variant of this reference modulation scheme could be used to control the differential synchronous phase transients, while reducing the klystron power requirements. In this scheme the differential is minimized, however there remain transient impacts on $V_{\text {cav }}$ such that it is modulated significantly in amplitude, 
so there would be concomitant variations in synchrotron tune for each bunch along the turn.

As shown in $[22,23]$ for the case of constant cavity voltage, the cavity phase modulation that minimizes the klystron power is given by

$$
\varphi(t)=-\Delta \omega_{\mathrm{opt}} \int_{t_{0}}^{t} \frac{i_{b}(u)-I_{D C}}{I_{D C}} d u+\varphi\left(t_{0}\right)
$$

where $I_{D C}$ is the average the beam current, and $i_{b}(t)$ is a scalar representing the beam current modulation. The constant $\varphi\left(t_{0}\right)$ is a "free" parameter as power does not depend on a constant phase shift of the cavity phase. But this must be constrained to keep all cavities in phase (for a given ring), and retain the collision point at the detector center. As shown in [24], $\Delta \omega_{\text {opt }}$ is the optimal cavity detuning given by

$$
\Delta \omega_{\mathrm{opt}}=-\frac{\omega_{\mathrm{rf}} R / Q F_{b} I_{D C} \sin \left(\phi_{b}\right)}{V_{\mathrm{cav}}}
$$

where $F_{b}$ is the relative bunch form factor [25], $\phi_{b}$ is the beam stable phase (electron machine convention), and $\omega_{\mathrm{rf}}$ is the rf angular frequency.

As shown in [23], the constraint on the cavity voltage results in excessive required power when the stable phase differs from $\pi / 2$. Therefore, a tradeoff exists between some modulation of the cavity voltage and the available rf power, for the electron ring. This tradeoff should be explored once the rf power budget is finalized.

The performance of this scheme depends on the ability to impose the reference voltage. The LLRF feedback system will need sufficient gain at the first revolution harmonic $\left(f_{\text {rev }} \approx 128 \mathrm{kHz}\right.$ ) to assure that the cavity voltage tracks the modulated reference. The OTFB will help since it adds gain at the revolution harmonics.

The optimal klystron power should be in the order of $255 \mathrm{~kW}$ for the electron ring ( $131 \mathrm{~kW}$ for the beam, $93 \mathrm{~kW}$ dissipated in the cavity wall, $31 \mathrm{~kW}$ of reflected power) and $242 \mathrm{~kW}$ for the ion ring (all of it reflected, since there is almost no power dissipated on the cavity and the beam). As an alternative approach, it might be possible to adjust rf station and beam parameters $\left(R / Q, I_{D C}, V_{\text {cav }}\right)$ to achieve the same modulation on both rings so that the bunches collide at the IP.

\section{A. Optimal phase modulation estimate}

Figure 16 shows the phase modulation that minimizes the klystron power for each ring, using the default rf station parameters.

One challenge with this approach is that if the beam loss is significant during the fill and asymmetric between the two rings, the reference phase modulation will have to be adjusted during the fill to keep the transients matched.

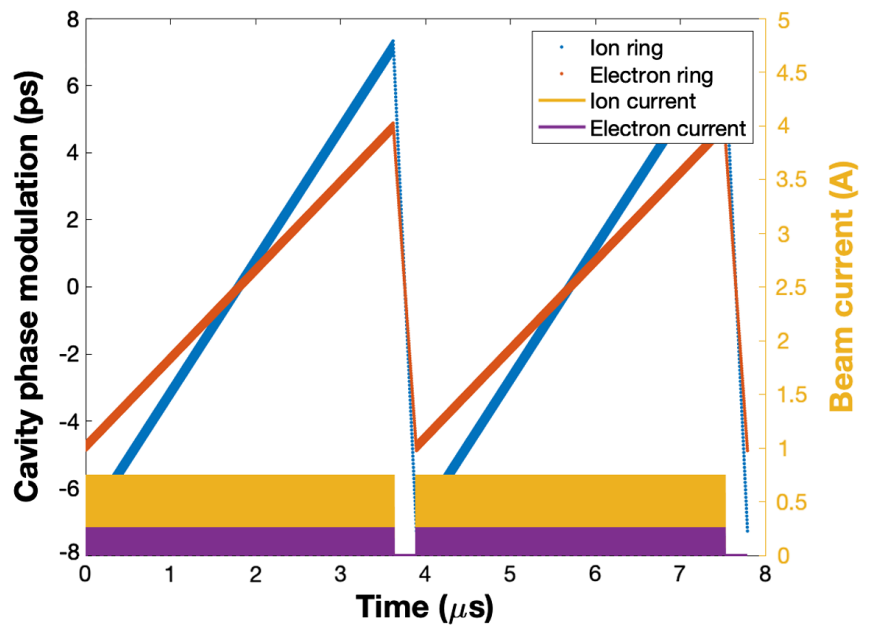

FIG. 16. Optimal phase modulation for both rings.

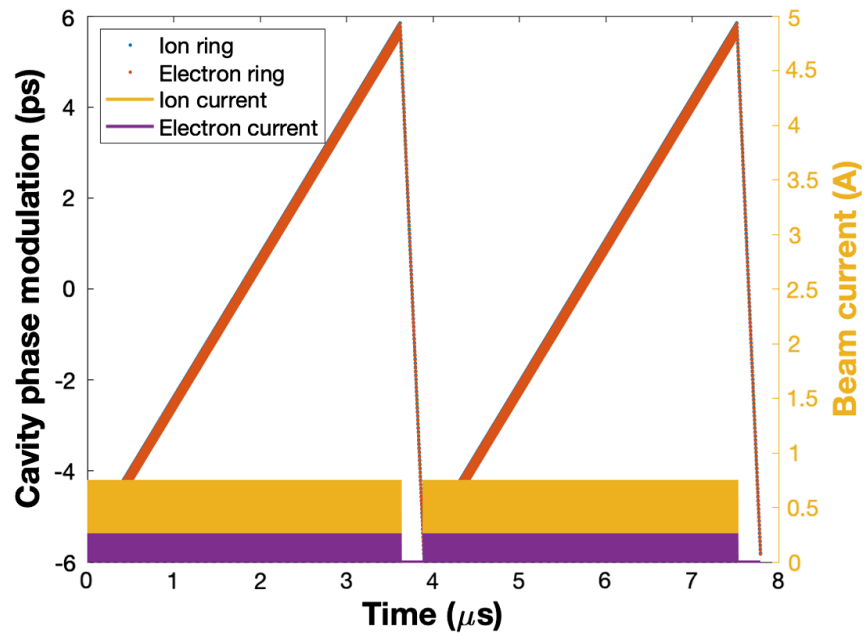

FIG. 17. Phase modulation with $R / Q$ adjustments.

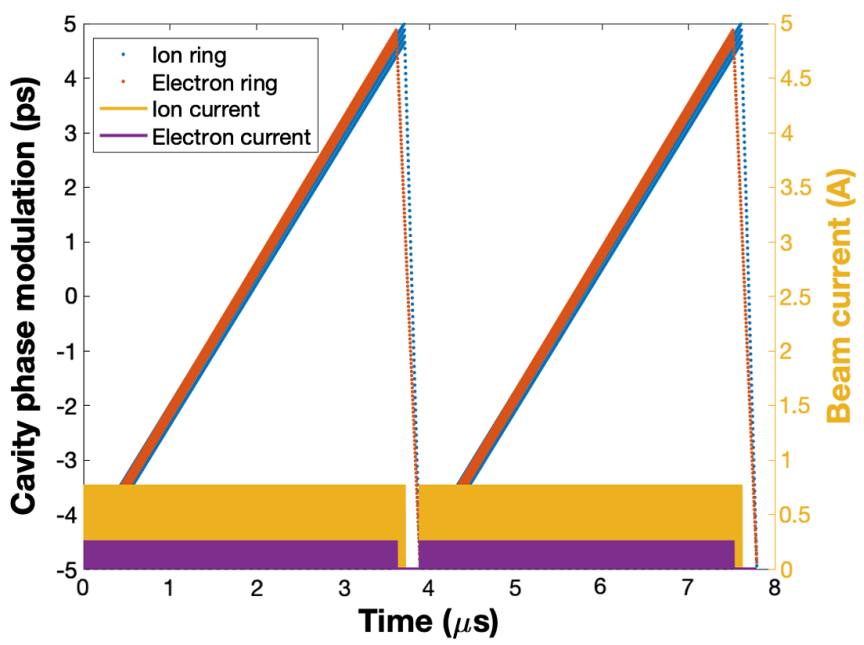

FIG. 18. Phase modulation with electron ring beam filling pattern adjustments. 


\section{B. Matching the two rings}

In the previous section, the phase references were adjusted with the goal of minimum klystron power. These modulations can also be applied to minimize the differential phase transient for the two rings through choices of $R / Q, I_{D C}$ (add bunches in the electron ring), or $V_{\text {cav }}$. A combination of these adjustments is of course possible too. They are investigated individually here though to develop some intuition on the range of the necessary changes through each approach.

As discussed in Sec. IV A, if the beam loss is significant and asymmetric between the two rings, rf parameters will have to be adjusted during the fill to keep the transients matched.

\section{C. $R / Q$ adjustments}

The phase modulation is proportional to $R / Q$, so the electron ring $R / Q$ should be increased and that for the ion ring should be reduced. An identical phase modulation is reached for an ion cavity $R / Q$ of 166 and an electron $R / Q$ of 259 (they were adjusted by 42), as shown in Figure 17. The change does not have to be symmetric, so different pairs of $R / Q$ values can be investigated. In fact, there is probably less flexibility on the electron ring $R / Q$ value.

\section{D. $V_{\text {cav }}$ adjustments}

The phase modulation is inversely proportional to $V_{\text {cav }}$. An identical phase modulation can also be achieved by a $\pm 22 \%$ change in the cavity voltage. A lower voltage will be needed for the electrons $(648 \mathrm{kV})$ and a higher for the ions (2.9 MV). This choice of operating points has an impact on the synchrotron tunes and the bunch lengths.

\section{E. Impose electron transient on the ion ring}

A modification of the cavity phase reference modulation scheme would involve imposing the electron ring gap transient on the ion ring phase reference. The electron ring transient could be continuously monitored and then used to actively adjust the modulation of the ion ring reference. This modified scheme has the added advantage that it is minimally affected by beam current changes due to beam loss and colliding particles, as it tracks any modulation or unique form of the electron ring phase transient, over time and uses this as an error signal to force the ion ring to follow symmetrically.

\section{F. Electron ring beam filling pattern adjustment}

The phase modulation has a linear relationship to the integral of the instantaneous current. It is thus possible to decrease the ion ring modulation by changing the ion ring beam filling pattern (reducing the gap length). Adding 11 bunches in the end of each train in the ion ring also results in very reasonable matching of the modulations, as shown in Figure 18. The feasibility of this scheme will depend on the rise time of the abort kicker. As mentioned in Sec. III, there is also a concern that any noncolliding bunches (e.g., the extra filled buckets in the ion ring) will not experience a beam-beam tune shift from colliding with a corresponding electron bunch, and these extra bunches will be at different betatron tunes (fragmenting the beam tune footprint), with potential lifetime reductions. In PEP-II this was attempted but had an operational impact of poor lifetime for the noncolliding bunches and was operationally challenging [27].

\section{G. IP time of collision shift}

All three adjustments from the methods above will result in approximately the same beam synchronous phase modulation, thus removing any modulation of the $z$ position of the collision point. On the other hand, the phase modulation will in turn lead to a modulation of the collision time with respect to the rf master oscillator. This shift is plotted in Fig. 19. This may have significance for any detector elements which need to measure time of flight relative to the rf oscillator rather than collision time. It should be noted though that the peak-to-peak excursion is about 12 ps, compared with an rms bunch length of 40 and 107 ps for the electron and ion ring respectively. Additionally, this shift is known and reproducible, so a modulated triggering signal could be generated for the detectors. The possible impact of this collision time shift vs bucket position on the crab cavity systems is discussed below.

\section{H. Effect on crab cavity systems}

The KEKB collider was the first machine to explore crab cavity systems as a means to increase luminosity $[28,29]$, and several facilities are exploring their potential use [30]. The KEKB $Q_{L}$ was $10^{8}$ [31]. Consistent with these examples, the EIC crab cavities would use a very

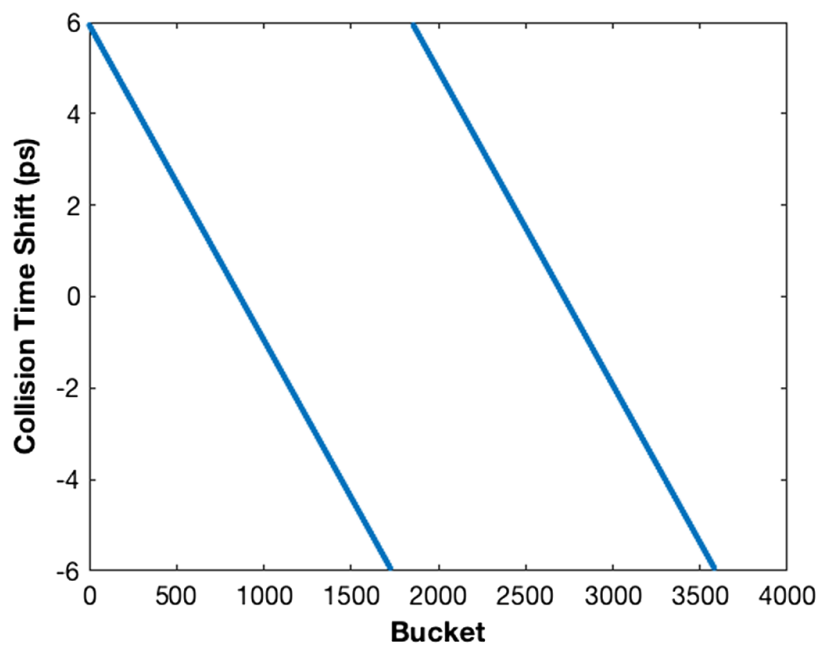

FIG. 19. Collision time shift due to reference phase modulation. 
high $Q_{L}$. As a result, it is not possible for the fields in the crab cavity to track the quick modulation imposed on the accelerating cavities without applying huge amounts of power. On the other hand, keeping the crab cavity voltage constant leads to loss of synchronization between the crab cavity and beam phase; and thus imperfect crabbing. For the HL-LHC, the modulation is small and the luminosity loss is acceptable [32]. Similar calculations should be conducted for the EIC as the machine conceptual design matures and estimates for the lattice functions and crabbing magnitude are available. Since the EIC bunch length may be much smaller than the HL-LHC, the luminosity loss will probably be even smaller than in the HL-LHC. The EIC situation is a bit more complicated though since the two rings will have different crabbing voltages, $\beta^{*}$ values at the crab cavity etc., so the same phase modulation could possibly lead to different transverse offsets for the colliding bunches.

\section{COUPLED-BUNCH INSTABILITIES}

The stability of the beams is critically determined by the impedance seen by the beam and the damping mechanisms present. The motion of the beam system is usually studied and represented by a set of even fill eigenmodes, which is a Fourier decomposition of the beam motion into normal modes. Effectively, the beam samples the impedance seen at the synchrotron sidebands around each rf harmonic, repeating this sampling going up to very high frequencies as determined by the bunch length. In the frequency domain, the stability of each mode $\ell$ is determined by the difference between driving and damping impedances, plus considerations of damping due to synchrotron radiation or the action of an external damping (feedback) system. The modal growth rates are given by:

$$
\sigma_{l} \approx-d_{r}+\frac{\eta q I_{D C}}{2 E_{o} T_{o} \omega_{s}} \mathcal{R}\left[\sum_{p=-\infty}^{\infty} \omega Z^{\|}(\omega) e^{-\sigma_{z}^{2} \omega^{2} / c^{2}}\right]
$$

where $\sigma_{l}$ is the growth rate of mode $\ell, Z^{\|}(\omega)$ is the cavity impedance, $d_{r}$ is the synchrotron radiation damping rate, $\eta$ is the slip factor, $T_{o}$ is the revolution period, $\omega_{s}$ is the synchrotron angular frequency, $c$ is the speed of light, $\sigma_{z}$ the bunch length, and $\omega=(p N+l) \omega_{\text {rev }}+\omega_{s}$, with $\omega_{\text {rev }}$ the ring revolution frequency and $N$ the number of bunches. These equations use the macroparticle model and apply to the electron ring. For the ion beam, these equations provide an approximation to the more exact expressions presented in $[33,34]$.

This summation over frequencies acts to fold all the impedance effects down into the single band of modes spanning half the rf frequency spaced by the revolution frequency. This sampling of impedances is a function of fill pattern, so that filling every bucket, or every other, or every third, etc. generates different unstable modal patterns [35], the modal structure of uneven fills has been used by Prabhakar to improve beam stability thresholds [36].

The driving impedances can be within the rf cavity bandwidth (due to the large impedance of the cavity fundamental), from higher order modes within the rf cavity, and also from external resonators in the ring (from vacuum chamber structures, etc.). Any source of impedance may drive beam instabilities.

The bare cavity fundamental impedance has a natural bandwidth (typically higher $Q$ for superconducting systems), but the action of the direct loop spreads this impedance out over a wider bandwidth. As such, and due to the low revolution frequencies of the EIC machines, the effective impedance of normal-conducting cavityLLRF systems (as used in PEP-II), can drive instabilities at many revolution harmonics (coupled-bunch modes). The action of the direct and one-turn (comb) feedback loops reduces the impedance seen by the beam at the synchrotron sidebands, helping reduce growth rates. The residual growth from impedances driving instabilities must then be controlled with feedback signals acting through the rf and LLRF systems and through dedicated broadband feedback kicker systems. It is possible to take advantage of the rf and LLRF system to implement the control on modes within the cavity bandwidth as these high power components have the greatest power within the necessary bandwidth [37].

Even superconducting if cavity systems as applied to high current machines with low revolution frequencies, can have coupled-bunch instabilities. Since the cavities will have a $Q_{\text {ext }}$ of $\approx 100,000$, whereas the revolution frequency is $\approx 128 \mathrm{kHz}$, they will mostly drive mode -1 . This can be mitigated via the action of the direct and one-turn delay feedback systems. Superconducting cavities also tend to have lower R/Q for HOMs.

Coupled-bunch instability control systems can mitigate the HOM driven instabilities with correction signals in a special wide-band kicker and power stage. Such systems have been used before in multiple machines [38-41].

The cavity and feedback models were used to estimate the open and closed loop cavity impedance for the EIC example implementations. Coupled-bunch instability growth rates driven by the cavity fundamental impedance were then computed for the uniform fill (every bucket) case. Figures 20, 21 show the results for the $10 \mathrm{GeV}$ electron ring $(0.26 \mathrm{~A})$ and the $200 \mathrm{GeV}$ ion ring $(0.75 \mathrm{~A})$ respectively. The synchrotron radiation damping rate $d_{r}$ is $156 \mathrm{~s}^{-1}$ for the electron ring and effectively zero for the ion ring at these energies.

These figures correspond to a simulation of a LLRF architecture with a direct feedback (with the delay and gains estimated earlier), but without a OTFB. The results suggest the electron ring is already stable in closed loop. The effectiveness of the impedance control through the direct loop would be reduced if the loop delay were 


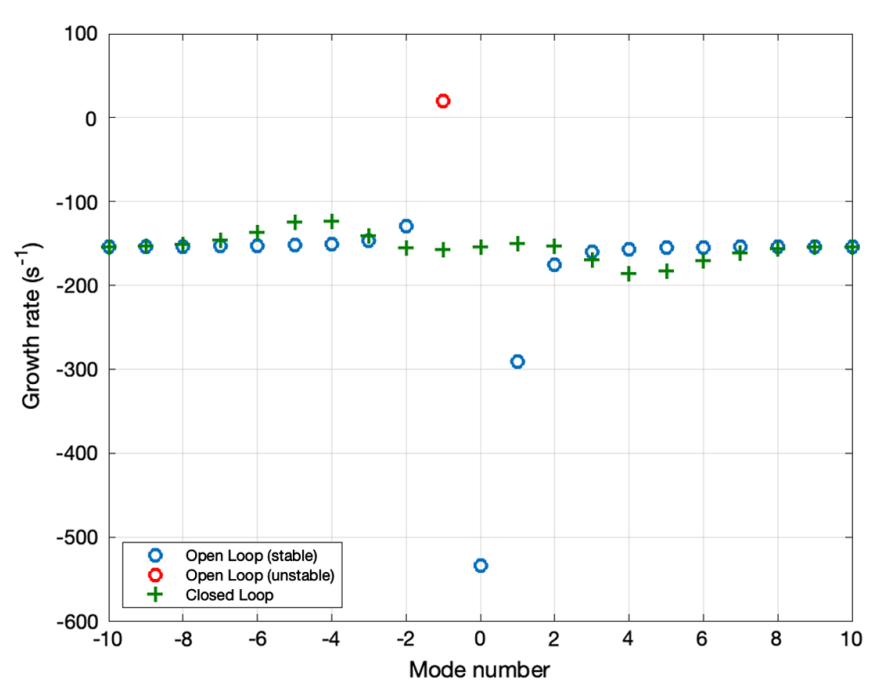

FIG. 20. Growth rate estimates for $10 \mathrm{GeV}$ electron ring.

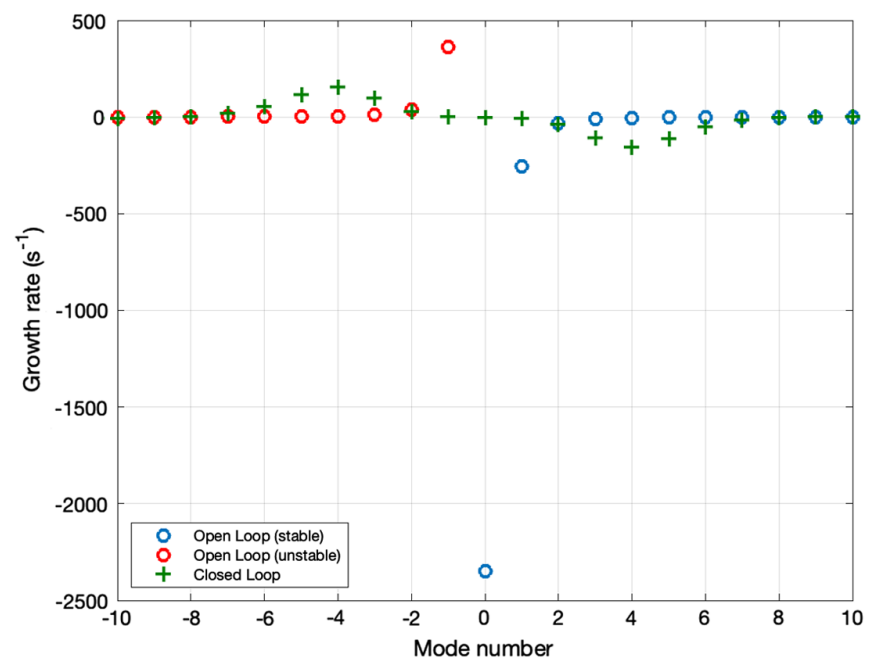

FIG. 21. Growth rate estimates for $200 \mathrm{GeV}$ ion ring.

increased, and this also must be studied as the machine conceptual design, with proposed klystron and cavity layouts, is maturing. The impact of a longer loop delay is investigated in Sec. VI.

For the ion ring, mode -4 has a slow positive growth rate of $155 \mathrm{~s}^{-1}$. This growth rate will be further reduced by a factor of 5 to 10 through the action of the one-turn feedback. The resulting growth rate $\left(16-31 \mathrm{~s}^{-1}\right)$ is orders of magnitude smaller than the synchrotron frequency of $6000 \mathrm{~Hz}$, so it can be easily controlled through a dedicated bunch-by-bunch longitudinal feedback system. Alternatively, stability can probably be achieved through manipulations of the LLRF parameters. Such a schemewhich exchanges loop stability margin for beam stabilitywas used in the last PEP-II run $[13,14]$. It should be noted that the growth rates for the modes outside the closed loop bandwidth do match the synchrotron radiation damping rates of 156 and $0 \mathrm{~s}^{-1}$ for the electron and ion ring respectively, as expected from Equation (3).

Similar studies are required for the lower energy and higher beam current EIC configurations. These configurations will be more challenging from a coupled-bunch instability perspective, since the beam current is higher, the energy is lower, and the synchrotron frequency is lower. All three factors lead to increased growth rates, so a careful sensitivity analysis on LLRF parameters should be conducted to determine stable operational points.

\section{IMPACT OF LOOP DELAY}

The studies in this paper have been based on a $320 \mathrm{~ns}$ loop delay, which is achievable if the rf power stages are located relatively close to the beam line components. This assumption is not appropriate if the facility design is based on an existing tunnel and civil engineering from an earlier machine, which did not incorporate rf feedback techniques, and where the rf power systems may have been located in a surface building for maintenance and operational reasons. This was the case for the PEP-II facility, which was constructed in the original PEP-I tunnel with existing surface rf power stage buildings and electrical feeds.

To explore the impact of the longer delays, we study the earlier case for the proposed electron ring, but with a $1000 \mathrm{~ns}$ loop delay. This study implements the direct loop but not the OTFB or feedforward. The gain of the direct loop is reduced proportionally to the increase in delay, to satisfy the direct loop's stability requirement. To compare the cases, we use the same gain-delay product in the two cases, so they have similar loop stability margins.

The impact of the reduced direct loop gain on growth rates is seen in Fig. 22. For the 320 ns case, all modes are very well damped, but for the longer delay mode -2 is marginally stable, and the stability of mode -1 is greatly

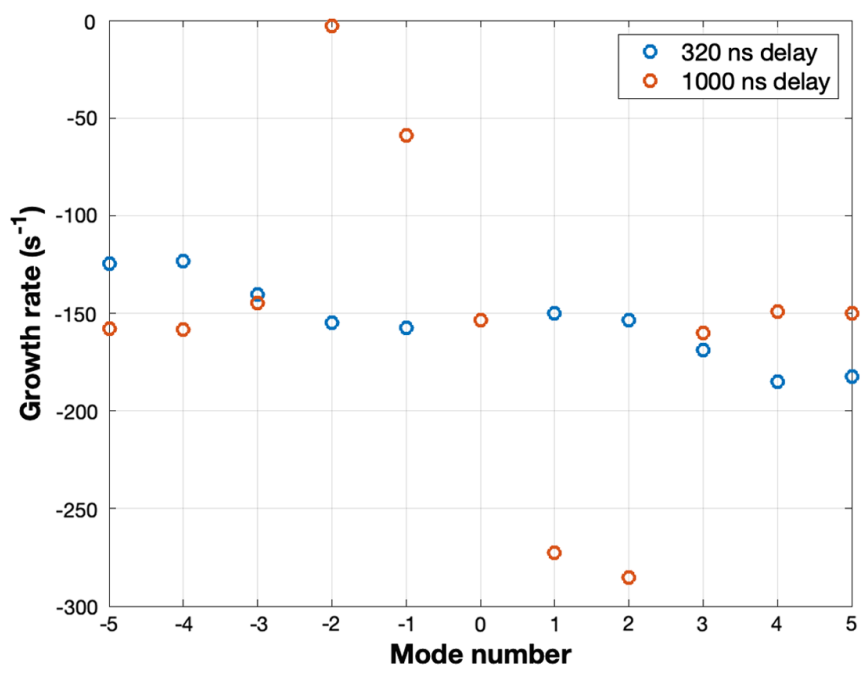

FIG. 22. Growth rate estimates for $10 \mathrm{GeV}$ electron ring for 320 and 1000 ns delays. 


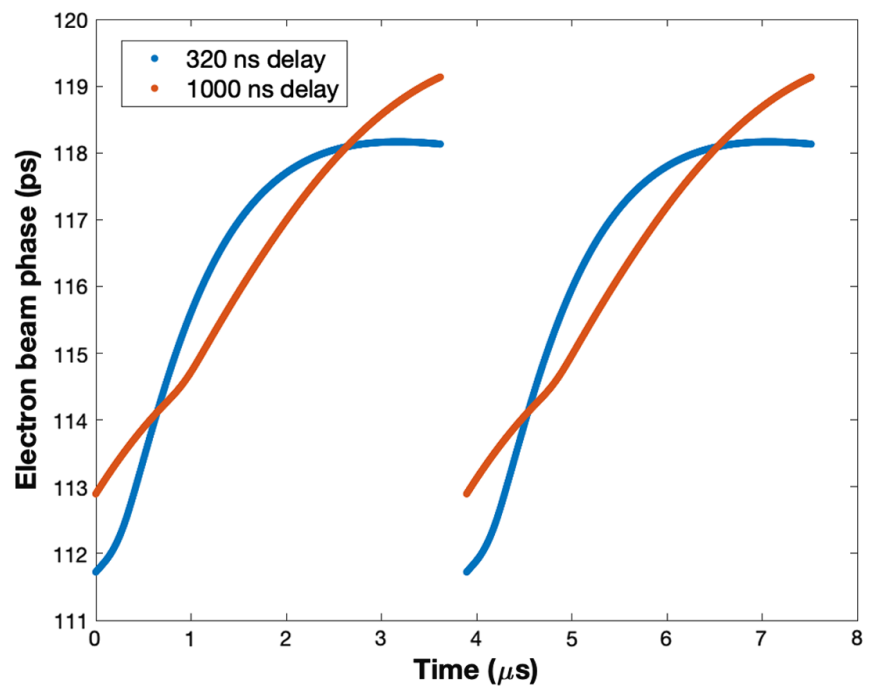

FIG. 23. Gap transient estimates for 320 and 1000 ns delays.

reduced as well. If the current were increased in this example the modes would become unstable. Figure 23 shows the delay impact on the gap transients for the nominal $0.26 \mathrm{~A}$ fill in the two train pattern used in earlier sections. The synchronous phase transients for the cases are not very different in amplitude, though they have different temporal characteristics reflecting the change in the transient response of the closed-loop system. Unlike the impact of the growth rates, the longer delay has smaller significance for the synchronous phase transients. The form of this transient would change more if the cavity filling time, and bunch train length, changed significantly.

\section{POTENTIAL COMPLICATIONS}

These various ideas which impose current variations among the bunches in a ring rely on the desired currents and bunch populations being maintained during injection and operation. But there are very real physical effects which might make the operation with double intensity bunches in some buckets very challenging. For example, Touschek scattering losses will be greater for these higher intensity bunches, so their lifetime will be lower. In the case of ideal top-up injection the desired current distribution would be maintained as currents are lost and restored. The achievable performance of the top up and injection system should be realistically estimated, and the simulations run with current distributions reflecting probable variations in operation. These current variations from realistic injections will impact the effectiveness of the various schemes resulting in shifts of the IP and modulations on the gap transients. It is likely that the operational lifetimes with losses out of some of the buckets will generate modulations in the gap transients and IP shifts.

The operation of any of these schemes assumes some consistent behavior of the power stages. Historically, the linearity and small-signal bandwidth of the LLRF and power (klystron) stages has been seen to have enormous impact on the effectiveness of the impedance control loops. In operation, the dynamic range of the signal path requires a large signal delivering power to the cavity and beam, while also providing faithful replication of the small sideband modulations at the synchrotron sidebands (which provide the impedance control). These small sidebands can be $80 \mathrm{~dB}$ or more below the carrier (power signal) level. If the klystron or other signal elements have nonlinear characteristics, the small-signal gain can be very different from the large-signal gain at the operating point. The nonlinear behavior of the various rf signals can easily fold information from an upper sideband to a lower, and vice versa [42]. The technical implementation of these signal processing systems can be very challenging in terms of required linearity and dynamic range. One challenge for the system designers is that very few of these system components are tested by manufacturers with the types of dynamic signals required for use in accelerator operation. The designers must do significant prototyping and testing to quantify possible performance of hypothetical architectures, and then use these simulation tools, with realistic imperfections included, to then estimate the likely performance of the accelerator. This requires skill and time to converge on a practical implementation [43].

Another practical consideration is that the physical system is composed of multiple rf stations, each with unique characteristics and imperfections. In operation, each station is uniquely configured to an operating point, and the behavior of each station is not identical. The superposition of all these unique system responses is what determines the beam stability, gap transients, noise, etc. There may be operational needs to run the accelerator with a parked rf cavity or with a klystron not delivering power. For these cases the remaining $\mathrm{rf}$ stations have to make up the necessary rf gap voltage, and the dynamics of the beam and stations will be different. Parking two identical unpowered cavity systems in perfect symmetry above and below the revolution harmonic cancels the impedances, but in real world systems the residual impedance from the parked cavities adds another periodic transient to the synchronous phase, which is again reflected in an IP shift. These operational situations must also be estimated and understood as part of the design and optimization of the accelerator. The operational management of such large complex systems, with many individual power systems each requiring unique configuration and care taking to maintain operational state should be considered in the design and cost estimation for the new facility.

\section{SUMMARY, CONCLUSIONS, AND FUTURE STEPS}

These initial studies give us confidence that solutions do exist for the example case. The developed tools can be 
adapted to evaluate various EIC machine designs and implementations. The methods used here are generally applicable to study more cases, and it is important to continue this evaluation as the EIC design evolves and various new $\mathrm{rf}$ configurations and ring parameters are proposed.

We have explored conventional LLRF feedback using well proven techniques such as OTFB, feedforward, and phase modulation, and the studies show acceptable phase transients and matching between the rings so that the bunches collide at the IP with some predictable periodic variation in arrival time and with acceptable klystron power. Fill pattern modulation offers the promise of almost complete cancellation of the transient for most of the turn and reduced klystron power, and has been demonstrated experimentally in several rings. How practical it will be on a daily basis and how well it can be maintained over a long store with two very different beams has yet to be determined, however with full energy top-off of the electron ring and flexible bunch formation schemes in the ion ring it will be worth considering in many cases.

Note that in this study the maximum number of cavities was installed in each ring, but the electron ring current was limited by synchrotron radiation power. There are other cases at lower energy but higher current where the electron ring transient is stronger. These cases should be studied in more detail in the future and may require the development of further new techniques to control the system.

More techniques exist to mitigate or match the gap transient effects. For example, the coupling factor and the cavity $Q_{L}$ can be adjusted to better match the transients in the two rings. These additional techniques will be investigated once the EIC design has matured.

The models to date do not include any realistic engineering-level imperfections and these impacts need to be estimated as part of the formal conceptual design report (CDR). For this baseline study, we have adopted conventional feedback schemes and sized the klystron power accordingly. Care will be needed to understand and control the coupled-bunch modes driven by the detuned fundamental mode impedance. Initial studies indicate that these growth rates should be similar to those seen in PEP-II and KEK-B and should be controllable by feedback systems, but more detailed studies are needed. The operation of the PEP-II LER was extremely challenging at the ultimate 3.2 A current, and this experience can guide the necessary simulation of a high-current EIC ring and LLRF system design. Understanding the tradeoffs between the stability of the LLRF feedback loops themselves, and the stability of the low-order coupledbunch beam modes is another topic required as part of the formal CDR effort [14]. The HOM driven instabilities are not studied in this paper, and these effects must be studied in the design and estimation of necessary all-mode coupled-bunch feedback systems.
The authors believe the most useful path forward to continue these studies is to develop a generally applicable tool set of longitudinal dynamics simulation models, and flexibly configurable LLRF models. A LLRF and rf system design tool set could be used to compare the evolving facility designs, help optimize the rf systems and operational performance, maximize luminosity and minimize rf power costs. As the LLRF topologies are explored, engineering-level models of LLRF functions, including realistic imperfections, nonlinearities, noise floors, etc. can be added to this tool set, and would be very valuable for the detailed system design and optimization of practical engineered systems. These tools would be of general use to the accelerator community in consideration of high current circular facilities of the future and upgrades to existing machines.

\section{ACKNOWLEDGMENTS}

The authors would like to thank Andrew Hutton and Dmitry Teytelman for many useful conversations, ideas, and suggestions. We also thank our PRAB reviewers who made many important suggestions which helped guide us to this submitted paper. This work is supported by the U.S. Department of Energy, Office of Science, Office of High Energy Physics, under Award No. DE-SC-0019287.

\section{APPENDIX: SIMULATION AND MODEL VERIFICATION}

The model and simulation were validated by comparing the system rf parameter sensitivity to theoretical expressions.

\section{Steady state}

To test the cavity, klystron, and coupler models in steady state, the klystron power was investigated as a function of cavity and coupler parameters (detuning and $Q_{L}$ ) for the electron ring. To achieve a steady state in the simulation, a uniform beam pattern current was used (no gap or structure in the fill). The number of particles per bunch was reduced to achieve the desired average beam current. As shown in [24], the generator current $I_{g}$ is given by

$$
\begin{aligned}
I_{g}= & {\left[\frac{V_{\mathrm{cav}}}{2(R / Q)}\left(\frac{1}{Q_{\mathrm{ext}}}+\frac{1}{Q_{0}}\right)+I_{D C} F_{b} \cos \left(\phi_{b}\right)\right] } \\
& -i\left[I_{D C} F_{b} \sin \left(\phi_{b}\right)+\frac{V_{\mathrm{cav}} \Delta f}{f_{\mathrm{rf}}(R / Q)}\right]
\end{aligned}
$$

where $Q_{\text {ext }}$ is the cavity external quality factor, $Q_{0}$ is the unloaded cavity quality factor, $\Delta f$ is the cavity detuning, and $f_{\text {rf }}$ is the rf frequency. The loaded cavity quality factor is given by 


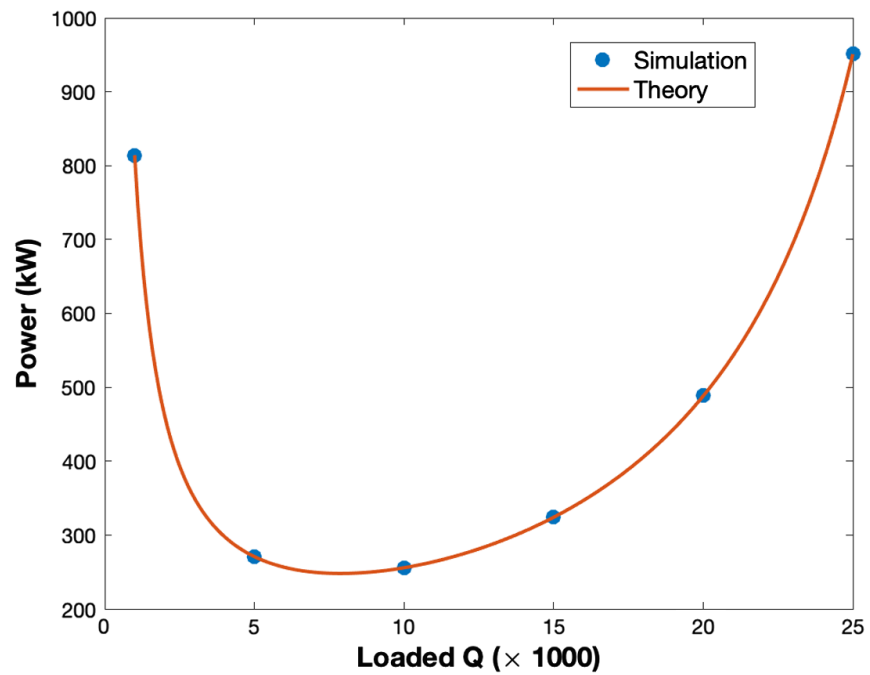

FIG. 24. Average klystron power with $Q_{L}$.

$$
\frac{1}{Q_{L}}=\frac{1}{Q_{\mathrm{ext}}}+\frac{1}{Q_{0}}
$$

The average klystron power is given by

$$
P_{g}=\frac{1}{2}(R / Q) Q_{\mathrm{ext}}\left|I_{g}\right|^{2} .
$$

The average klystron power dependence on $Q_{L}$ is shown in Fig. 24 for a detuning of zero, an $R / Q$ of 217, $V=790 \mathrm{kV}, I_{D C}=0.26 \mathrm{~A}$, and $F_{b}=0.9907$. There is very good agreement between the simulation and theory. The optimal power of $248 \mathrm{~kW}$ is achieved for a $Q_{L}$ of 9,100 .

The cavity detuning was then manually adjusted in the model. The tuner loop, which automatically adjusts detuning to minimize peak klystron power, was switched off. The $Q_{L}$ was set to 5,200. Using Eq. (A2) it is possible to calculate the average klystron power for different detuning levels. Figure 25 compares the simulated and calculated average klystron power, again showing good agreement. Since the beam loading over a turn is low and constant, there is not a significant dependence on the detuning.

From [24], the optimal cavity detuning $\Delta f_{\text {opt }}$ which minimizes the average klystron power is given by

$$
\Delta f_{\mathrm{opt}}=-\frac{f_{\mathrm{rf}} R / Q F_{b} I_{D C} \sin \left(\phi_{b}\right)}{V_{\mathrm{cav}}}
$$

The optimal detuning for the parameters above is $-12.9 \mathrm{kHz}$, in agreement with the result shown in Fig. 25.

\section{Transient behavior (beam loading)}

The studies above validate the steady state behavior of the model and the accurate depiction of the klystron, cavity, and coupler. To validate the transient behavior of the model

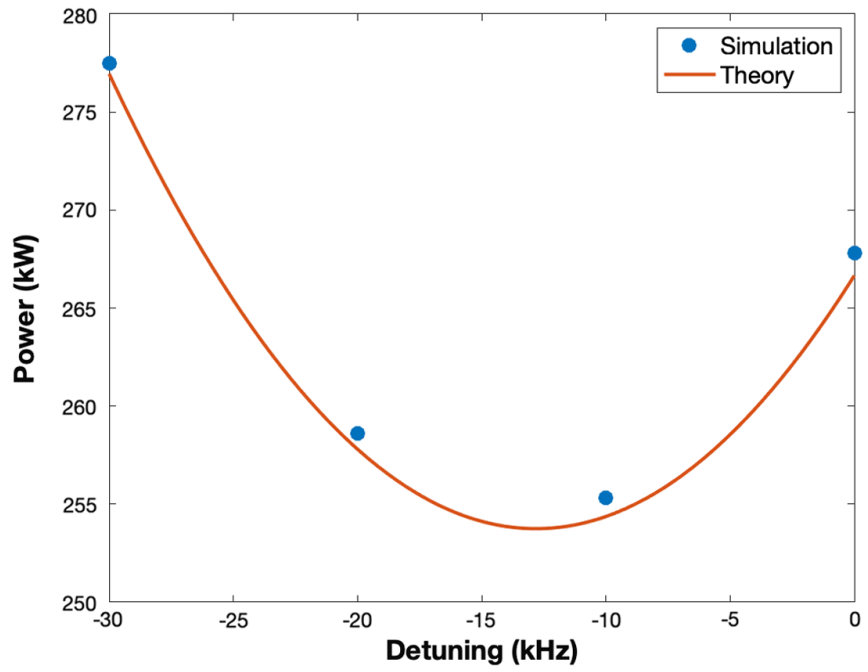

FIG. 25. Average klystron power with detuning.

as well, a single bunch train of 432 bunches at $1.467 \times 10^{10}$ electrons per bunch was then injected into the cavity in the absence of feedback. The klystron was set at a constant level to maintain the desired cavity voltage. For this study the beam does not circulate, instead a single transient for the cavity-rf system is excited by the bunch train. The simulation continues in time to capture the full transient, allowing the cavity to return to equilibrium.

For a detuning of zero, the steady state induced voltage (i.e., for a uniform beam pattern) is given by

$$
V_{\text {ind }}=I_{\text {peak }} F_{b}(R / Q) Q_{L} e^{-j \phi_{b}}
$$

where $I_{\text {peak }}$ is the rf component of the instantaneous beam current.

Therefore, the cavity voltage after the arrival of a bunch train is

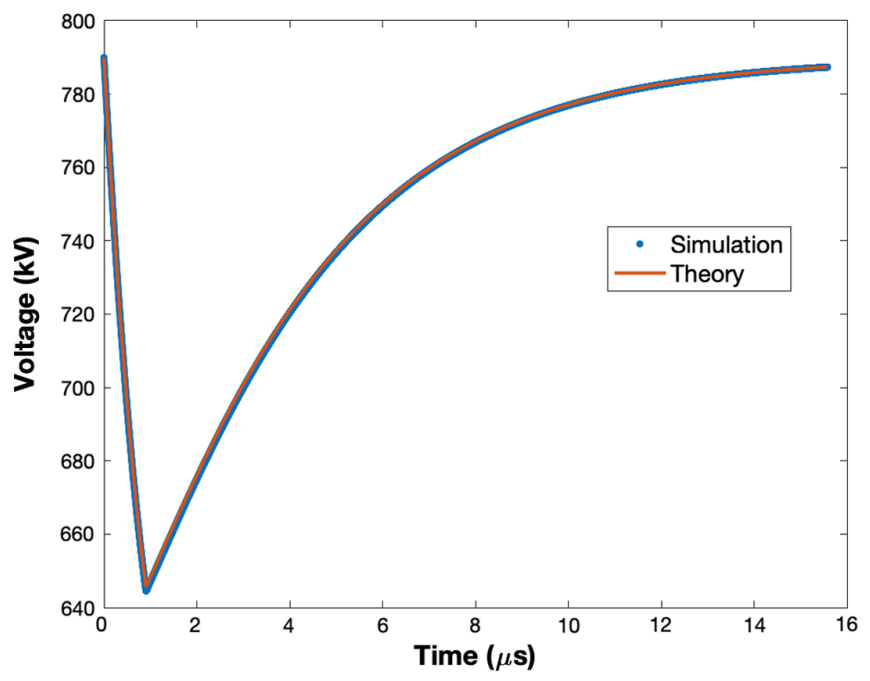

FIG. 26. Cavity voltage transient due to beam loading. 


$$
V_{\text {cav }}(t)=V_{o}+V_{\text {ind }}-\left(V_{\text {ind }}\right) \exp ^{-t / \tau_{F}}
$$

where $\tau_{F}=Q_{L} /\left(\pi f_{\text {rf }}\right)$ is the cavity filling time. The cavity voltage returns to its nominal value with the same time constant $\tau_{F}$.

Figure 26 shows these theoretical expressions and the simulated cavity voltage. Once again, there is very good agreement between theory and simulation.

[1] P. Baudrenghien, Low-level rf systems for synchrotrons. Part II: High intensity. Compensation of beam-induced effects, in CERN Accelerator School Course on RF Engineering (CERN, Geneva, Switzerland, 2000), pp. $175-209$.

[2] T. Kobayashi and K. Akai, LLRF controls including gap transients at KEKB and plans for SuperKEKB, in 58th ICFA Advanced Beam Dynamics Workshop on High Luminosity Circular $e^{+} e^{-}$Colliders (JACoW Publishing, Geneva, Switzerland, 2017), p. WET2H7.

[3] F. Pedersen, Beam loading effects in the CERN PS Booster, IEEE Trans. Nucl. Sci. 22, 1906 (1975).

[4] F. Pedersen, RF cavity feedback, in B Factories: The State of the Art in Accelerators, Detectors, and Physics (Stanford, CA, 1992), pp. 192-207.

[5] K. Hirosawa, K. Akai, E. Ezura, T. Kobayashi, K. Nakanishi, and S.-i. Yoshimoto, Advanced damper system with a flexible and fine-tunable filter for longitudinal coupled-bunch instabilities caused by the accelerating mode in SuperKEKB, Nucl. Instrum. Methods Phys. Res., Sect. A 953, 163007 (2020).

[6] D. Boussard, Control of cavities with high beam loading, IEEE Trans. Nucl. Sci. 32, 1852 (1985).

[7] Y. Zhang, JLEIC: A High Luminosity Polarized ElectronIon Collider at Jefferson Lab, J. Phys. Conf. Ser. 1350 (2019).

[8] A. Seryi et al., The US Electron Ion Collider Accelerator Designs, in Proceedings, North American Particle Accelerator Conference (NAPAC 2019): Lansing, MI, USA, September 2019 (JACoW Publishing, Geneva, Switzerland, 2020), p. MOOHC2.

[9] Y. Zhang, JLEIC: A High Luminosity Polarized ElectronIon Collider at Jefferson Lab (2019), North American Particle Accelerator Conference (JACoW Publishing, Geneva, Switzerland, 2020): Lansing, MI, USA, September 2019.

[10] R. Tighe and P. Corredoura, RF feedback simulation results for PEP-II, Conf. Proc. C 950501, 2666 (1996).

[11] R. Tighe, PEP-II RF feedback system simulation, in International Workshop on Collective Effects and Impedance for B Factories (CEIBA 95) (1995), pp. 445-455 [Report No. KEK-PROCEEDINGS-96-6, Tsukuba, Japan].

[12] R. Tighe, RF feedback simulation for the PEP-II B factory, Conf. Proc. C 940627, 1957 (1994).

[13] C. Rivetta, T. Mastorides, J. D. Fox, D. Teytelman, and D. Van Winkle, Modeling and simulation of longitudinal dynamics for low energy ring-high energy ring at the positron-electron project, Phys. Rev. ST Accel. Beams 10, 022801 (2007).

[14] T. Mastorides, C. Rivetta, J. D. Fox, D. VanWinkle, and D. Teytelman, Analysis of Longitudinal Beam Dynamics Behavior and RF System Operative Limits at High Beam Currents in Storage Rings, Phys. Rev. Accel. Beams 11, 062802 (2008).

[15] T. Mastorides, C. Rivetta, J. D. Fox, D. V. Winkle, and P. Baudrenghien, RF system models for the CERN Large Hadron Collider with application to longitudinal dynamics, Phys. Rev. Accel. Beams 13, 102801 (2010).

[16] D. Boussard, Design of a ring RF system, in CERNRutherford Accelerator School: RF Engineering for Particle Accelerators (CERN, Geneva, Switzerland, 1991), pp. 0294-322.

[17] P. Baudrenghien and G. Lambert, Reducing the impedance of the travelling wave cavities feed-forward and one turn delay feed-back, in 10th Workshop on LEP-SPS Performance: Chamonix X (2000), pp. 94-101.

[18] J. M. Byrd, S. De Santis, J. Jacob, and V. Serriere, Transient beam loading effects in harmonic RF systems for light sources, Phys. Rev. Accel. Beams 5, 092001 (2002).

[19] H. Wang, J. Dai, Q. Qin, R. Rimmer, D. Teytelman, S. Wang, J. Xing, J. Yue, and Y. Zhang, Transient beam loading due to the bunch train gap and its compensation experiments at BEPC-II and ALS, J. Phys. Conf. Ser. 1067 (2018).

[20] D. Teytelman, Transient beam loading (2017), FCC Week 2017, https://indico.cern.ch/event/556692/contributions/ 2590414.

[21] W. Ross, R. Claus, and L. Sapozhnikov, Gap voltage feed forward module for PEP-II low level RF system, in 17th IEEE Particle Accelerator Conference (PAC 97): Accelerator Science, Technology and Applications Vancouver, British Columbia, Canada, May 12-16, 1997 (JACoW Publishing, Geneva, Switzerland, 1997).

[22] T. Mastoridis, P. Baudrenghien, and J. Molendijk, Cavity voltage phase modulation to reduce the high-luminosity large hadron collider rf power requirements, Phys. Rev. Accel. Beams 20, 101003 (2017).

[23] I. Karpov and P. Baudrenghien, Transient beam loading and rf power evaluation for future circular colliders, Phys. Rev. Accel. Beams 22, 081002 (2019).

[24] J. Tückmantel, Cavity-beam-transmitter interaction formula collection with derivation, Tech. Report No. CERN-ATS-Note-2011-002 TECH, 2010.

[25] In this work, the relative bunch form factor $F_{b}$ is defined as in [26]. With this definition, $F_{b}$ is equal to 1 for infinitely short bunches.

[26] J. Tückmantel, Adaptive rf transient reduction for high intensity beams with gaps, Tech. Report No. CERN-AB2006-030, 2006.

[27] M. Sullivan and U. Weinands (private communication).

[28] Y. Funakoshi (KEKB Commissioning Group), Operational experience with crab cavities at KEKB, in ICFA MiniWorkshop on Beam-Beam Effects in Hadron Colliders (CERN, Geneva, Switzerland, 2014), pp. 27-36, arXiv: 1410.4036 . 
[29] K. Akai et al., Commissioning and Beam Operation of KEKB Crab RF System, in 13th International Workshop on RF Superconductivity (Beijing Univ., Beijing, China, 2007).

[30] R. Calaga, O. Capatina, and G. Vandoni, The SPS Tests of the HL-LHC Crab Cavities, in Proc. 9th International Particle Accelerator Conference (IPAC'18), Vancouver, BC, Canada, April 29-May 4, 2018, International Particle Accelerator Conference No. 9 (JACoW Publishing, Geneva, Switzerland, 2018), pp. 846-849, https:// doi.org/10.18429/JACoW-IPAC2018-TUPAF057.

[31] K. Akai and Y. Funakoshi, Beam loading issues and requirements for the KEK B crab RF system, Conf. Proc. C 960610, 2118 (1996).

[32] E. Yamakawa, R. Apsimon, P. Baudrenghien, R. Calaga, and A. Dexter, Luminosity reduction caused by phase modulations at the hl-lhc crab cavities, Nucl. Instrum. Methods Phys. Res., Sect. A 908, 338 (2018).

[33] A. Chao, Physics of Collective Beam Instabilities in HighEnergy Accelerators (Wiley, New York, USA, 1993).

[34] P. Baudrenghien and T. Mastoridis, Fundamental cavity impedance and longitudinal coupled-bunch instabilities at the High Luminosity Large Hadron Collider, Phys. Rev. Accel. Beams 20, 011004 (2017).

[35] S. Prabhakar, New diagnostics and cures for coupled bunch instabilities, Ph.D. thesis, Stanford U., 2000.

[36] S. Prabhakar, J. D. Fox, and D. Teytelman, Curing Coupled Bunch Instabilities with Uneven Fills, Phys. Rev. Lett. 86, 2022 (2001).

[37] D. Teytelman, L. Beckman, D. Van Winkle, J. Fox, and A. Young, Development and testing of a low group-delay Woofer channel for PEP-II, in Proceedings of the 9th
European Particle Accelerator Conference, Lucerne, 2004 (EPS-AG, Lucerne, 2004), https://cds.cern.ch/record/ 794944.

[38] S. Khan, T. Knuth, J. Fox, S. Prabhakar, D. Teytelman, A. Young, G. Stover, A. Drago, and M. Serio, Commissioning of the BESSY-II longitudinal feedback system, in Proceedings of the European Particle Accelerator Conference, Vienna, 2000 (EPS, Geneva, 2000), pp. 1903-1905.

[39] J. Fox, H. Hindi, R. Larsen, S. Prabhakar, D. Teytelman, A. Young, A. Drago, M. Serio, and G. Stover, Multibunch longitudinal dynamics and diagnostics via a digital feedback system at PEP-II, DAPHNE, ALS and SPEAR, in Proceedings of the 6th European Particle Accelerator Conference, Stockholm, 1998 (IOP, London, 1998), pp. 296-298.

[40] T. Obina, W. X. Cheng, T. Honda, and M. Tobiyama, Longitudinal feedback system for the photon factory, in Proceedings of the 22nd Particle Accelerator Conference, PAC-2007, Albuquerque, NM (IEEE, New York, 2007), pp. 233-235.

[41] M. Bakr, H. Huck, M. Honer, S. Khan, R. Molo, A. Nowaczyk, A. Schick, P. Ungelenk, and M. Zeinalzadeh, Bunch-by-bunch feedback systems at the DELTA Storage Ring, Conf. Proc. C 1205201, 807 (2012).

[42] J. D. Fox, T. Mastorides, C. H. Rivetta, and D. Van Winkle, Selecting rf amplifiers for impedance controlled LLRF systems - nonlinear effects and system implications, Conf. Proc. C 070625, 2451 (2007).

[43] J. Fox, T. Mastorides, C. Rivetta, D. Van Winkle, and D. Teytelman, Lessons learned from positron-electron project low level rf and longitudinal feedback, Phys. Rev. Accel. Beams 13, 052802 (2010). 